\title{
Aspectos sociodemográficos e qualidade de vida de pessoas com Traumatismo da medula espinal
}

\author{
Sociodemographic Aspects and Quality of Life of people \\ with Spinal Cord Injury
}

\author{
Aspectos Sociodemográficos y Calidad de Vida de las \\ Personas con Traumatismo de Cable Espinal
}

\section{Camila Ferreira de Assis Soares ${ }^{1}$, Paulo Henrique Muleta Andrade ${ }^{2}$, Karla de Toledo Cândido Muller ${ }^{3}$, Serginaldo José dos Santos ${ }^{4}$}

\begin{abstract}
1.Fisioterapeuta no Centro Especializado em Reabilitação no setor de neurologia, Graduada pela Universidade Católica Dom Bosco (UCDB), Campo Grande-MS, Brasil.

2.Fisioterapeuta Doutor no Hospital Universitário Maria Aparecida Pedrossian- HUMAP, Coordenador do Centro Especializado em Reabilitação - CER IV, Campo Grande-MS, Brasil.

3.Professora Doutora na Universidade Católica Dom Bosco- UCDB, Campo Grande-MS, Brasil.

4.Professor Mestre na Universidade Católica Dom Bosco - UCDB, Campo Grande-MS, Brasil.
\end{abstract}

\begin{abstract}
Resumo
Introdução. O presente estudo aborda os aspectos sociodemográficos e epidemiológicos e sua correlação com a qualidade de vida das pessoas com traumatismo da medula espinal em processo de reabilitação física em um Centro Especializado em Reabilitação. Objetivo. Verificar e correlacionar variáveis sociodemográficas de pessoas com traumatismo da medula espinal com sua qualidade de vida. Método. Foram entrevistadas 25 pessoas, coletou-se seus dados sociodemográficos por meio de um questionário semiestruturado e aplicou-se a escala da ASIA e o instrumento geral da OMS-WHOQOL-bref. Resultados. Houve predomínio de homens adultos jovens, casados, renda baixa, a maioria com sequelas em paraplegia incompleta, entre 1 e 3 anos de lesão. A qualidade de vida geral foi avaliada como regular para $72 \%$ dos entrevistados, mantida nos domínios físico, psicológico e de meio ambiente, com melhor pontuação no domínio social prevaleceu considerado como bom. Não houve correlação entre as variáveis sociodemográficas e epidemiológicas, assim como com a Escala da ASIA e a qualidade de vida. Conclusão. Conclui-se que a qualidade de vida das pessoas com traumatismo da medula espinal foi percebida como regular, sem correlação com a idade do paciente, funcionalidade e tempo da lesão.
\end{abstract}

Unitermos. Traumatismos da Medula Espinal; Centros de Reabilitação; Reabilitação; Qualidade de vida

\footnotetext{
Abstract

Introduction. The present study deals with the sociodemographic and epidemiological aspects and their correlation with the quality of life of people with spinal cord injury in a physical rehabilitation process at a Specialized Rehabilitation Center. Objective. To verify and correlate sociodemographic variables of people with spinal cord injury with their quality of life. Method. 25 people were interviewed, their socio-demographic data was collected through a semi-structured questionnaire and the ASIA scale and the WHO-WHOQOL-bref general instrument were applied. Results. There was a predominance of young married men low low income, mostly with sequelae in incomplete paraplegia, between 1 and 3 years of injury. The overall quality of life was assessed as regular for $72 \%$ of respondents, maintained in the physical, psychological and environmental domains, with best score in the social domain prevailed considered as good. There was no correlation between sociodemographic and epidemiological variables, as well as the ASIA Scale and quality of life. Conclusion. It was concluded that the quality of life of people with perceived spinal cord injury was perceived as regular, without correlation with the patient's age, function and time of injury.
}

Keywords. Spinal cord injury; Rehabilitation centers; Rehabilitation; Quality of life 


\section{Resumen}

Introducción. El presente estudio aborda los aspectos sociodemográficos y epidemiológicos y su correlación con la calidad de vida de las personas con trauma de la médula espinal que se someten a rehabilitación física en un Centro de rehabilitación especializado. Objetivo. Verificar y correlacionar las variables sociodemográficas de las personas con trauma de la médula espinal con su calidad de vida. Método. se entrevistó a 25 personas, se recopilaron sus datos sociodemográficos a través de un cuestionario semiestructurado y se aplicaron la escala ASIA y el instrumento general WHO-WHOQOL-bref. Resultados. Predominaron los hombres adultos jóvenes, casados y de bajos ingresos, la mayoría con secuelas en paraplejia incompleta, entre 1 y 3 años de lesión. La calidad de vida general se evaluó como regular para el $72 \%$ de los encuestados, mantenida en los ámbitos físico, psicológico y ambiental, con una mejor puntuación en el dominio social, que se consideró bueno. No hubo correlación entre las variables sociodemográficas y epidemiológicas, así como con la escala ASIA y la calidad de vida. Conclusión. Se concluye que la calidad de vida de las personas con trauma de la médula espinal se percibió como regular, sin correlación con la edad del paciente, la funcionalidad y el tiempo de la lesión.

Palabras clave. lesiones de la médula espinal; centros de rehabilitación; rehabilitación; calidad de vida

Trabalho realizado no Centro Especializado em Reabilitação- CER, Campo Grande-MS, Brasil.

\section{INTRODUÇÃO}

O aumento de acidentes automobilísticos associados a violência tem elevado a incidência de traumas (80\%), incluindo o traumatismo da medula espinhal (TME), entre as causas não traumáticas (20\%) destacam-se os tumores, infecciosas, vasculares e degenerativas, constituindo-se em um importante problema de saúde pública. No Brasil a incidência de TME é cerca de 6 mil novos casos por ano, sendo o sexo masculino predominante, na proporção de 4:1, na faixa etária de 15 a 40 anos $^{1}$.

Os indivíduos com TME têm perda parcial ou total da função motora, sensitiva, autônoma e complicações em seu sistema orgânico. A gravidade do quadro depende do nível da lesão e do grau de dano da via aferente e/ou eferente ${ }^{2}$. 
De acordo com o nível medular lesionado o indivíduo pode tornar-se paraplégico ou tetraplégico. A tetraplegia resulta em perda da função dos membros superiores, bem como do tronco, membros inferiores e órgãos pélvicos. Já a paraplegia refere-se à perda de funções motoras e/ou sensoriais de segmentos torácicos, lombares e sacrais da medula espinhal, com a preservação dos membros superiores ${ }^{2,3}$.

Além das perdas motoras e sensoriais pode apresentar complicações, como: a dor neuropática, que se caracteriza por sensação desconfortável em queimação, em região na qual há perda ou diminuição da sensibilidade; alterações músculo-esqueléticas como ossificação heterotópica, que é a formação de osso em tecidos moles em locais onde normalmente não existe e a osteoporose (declínio na densidade mineral óssea). Quanto as alterações vasculares podem-se encontrar a trombose venosa profunda sendo decorrente da hipercoagulabilidade sanguínea, das alterações endoteliais e da estase venosa; hipotensão postural e disreflexia autonômica. A bexiga neurogênica, intestino neurogênico, úlceras por pressão e a espasticidade são outras alterações encontradas ${ }^{3}$. As complicações supracitadas, quando somadas, podem gerar incapacidades funcionais significativas, como para a realização das atividades de vida diária (AVDs) e reduzir sua qualidade de vida.

A reabilitação multidisciplinar desses pacientes deve ser feita desde o hospital até o domicílio. O atendimento em 
geral é com fisioterapia neurofuncional, respiratória e especialistas em próteses e órteses, terapeutas ocupacionais, psicólogos, fonoaudiólogos e assistentes sociais. A fisioterapia tem importante papel na manutenção de amplitudes de movimentos, fortalecimento muscular, aprimorar e promover a independência funcional do paciente. Sendo assim, deve ser garantida a assistência fisioterapêutica especializada a todos os indivíduos com TME, em especial durante o primeiro ano após a lesão ${ }^{3,4}$.

A relevância de se conhecer o perfil sociodemográfico e epidemiológico possibilita compreender melhor os quadros nosológicos e encontrar maneiras de diminuir/evitar possíveis complicações, possibilitando a melhora ou manutenção da qualidade de vida do paciente, ao formular estratégias diante das dificuldades encontradas dentro das singularidades de cada caso.

O objetivo deste estudo é verificar e correlacionar variáveis sociodemográficas de pessoas com traumatismo da medula espinhal com sua qualidade de vida.

\section{MÉTODO}

\section{Amostra}

Trata-se de um estudo transversal descritivo, não probabilístico com amostra por julgamento. Foram incluídos todos os pacientes maiores de 18 anos com traumatismo da medula espinhal (TME), em processo de reabilitação física em um Centro Especializado em Reabilitação (CER) na cidade 
de Campo Grande-MS, o qual é referência para a rede de atenção à saúde da pessoa com deficiência física e intelectual de seu território. O critério de exclusão foram os pacientes que apresentaram algum tipo de transtorno mental e não conseguiram responder o questionário e/ou não tiveram acompanhantes.

O projeto foi submetido ao Comitê de Ética de Pesquisa no: 62225616.2.0000.5162, foi apresentado o Termo de Consentimento Livre e Esclarecido (TCLE) aos pacientes, onde foi assinado pelo paciente e o pesquisador, após o sujeito ser esclarecido sobre quem seriam os pesquisadores, os objetivos e benefícios da pesquisa, assegurado o sigilo, a privacidade e o direito de opinar, em qualquer momento da investigação.

\section{Procedimento}

Realizou-se coleta dos dados por meio de um questionário semiestruturado, composto pelo perfil clínico e perguntas relacionadas ao momento e o atendimento após o trauma, além da investigação das variáveis sociodemográficas, condições de saúde e aspectos funcionais das principais atividades de vida diária (AVDs), possibilitando assim conhecer os principais problemas decorrentes da lesão. Quando o paciente e/ou acompanhante não souberam responder sobre algumas questões utilizou-se os prontuários da instituição.

A escala da "International Standards for NeurologicalClassificationofSpinal Cord Injury", 
popularmente conhecida por ASIA $(2015)^{5}$, foi aplicada para classificar quanto ao tipo de lesão (se completa ou incompleta) e determinar o nível neurológico, além de gerar um escore baseado nos achados sensitivos e motores. $\mathrm{Na}$ sequência e por fim, foi estudada a qualidade de vida utilizando-se o instrumento geral da OMS-WHOQOL-BREF $(\mathrm{OMS})^{6}$, norteado pelo estudo de Bampi, Guilhem e Lima7.

\section{Análise Estatística}

Os dados coletados foram trabalhados por meio de estatísticas descritivas e apresentados por meio de gráficos e tabelas, as variáveis qualitativas foram apresentadas em frequência relativa (n) e absoluta (\%) e para variáveis quantitativas em média \pm desvio padrão.

As análises de correlação foram calculadas pelo coeficiente de correlação de Spearman com o nível de significância de $5 \%(p<0,05)$.

\section{RESULTADOS}

Durante o período do estudo, foram entrevistados 25 pacientes no período de janeiro a março de 2017, dos quais 17 eram do gênero masculino (68\%) e 8 do gênero feminino (32\%).

As variáveis sociodemográficas mostrando as frequências absolutas e relativas dos participantes está representada na Tabela 1. 
Tabela 1. População do estudo segundo as variáveis sociodemográficas.

\begin{tabular}{ccc}
\hline Variação & Frequência absoluta (n) & Frequência relativa (\%) \\
\hline Gênero & 8 & \\
Feminino & 17 & 32 \\
Masculino & & 68 \\
Faixa etária & 9 & 36 \\
$18-28$ & 7 & 28 \\
$29-39$ & 5 & 20 \\
$40-50$ & 1 & 4 \\
$51-61$ & 3 & 12 \\
$62-72$ & 10 & 40 \\
Escolaridade & 13 & 52 \\
Ensino fundamental & 2 & 8 \\
Ensino médio & & \\
Ensino Superior & 12 & 48 \\
Renda & 6 & 24 \\
1 salário & 5 & 20 \\
2 salários & 2 & 8 \\
3 salários & & \\
4 salários & &
\end{tabular}

A idade variou de 18 a 72 anos, sendo que a média de idade foi de $37,2 \pm 14,60$ anos, destacando-se que a faixa etária mais acometida foi a de adultos jovens entre 18-28 anos (36\%), demonstrando a maior proporção de pessoas acometidas nesta faixa etária.

De acordo com os dados levantados $52 \%$ dos pacientes que sofreram TME tem ensino médio, $40 \%$ ensino fundamental e apenas $8 \%$ com ensino superior completo. $E$ como o estudo mostra, a prevalência é de pessoas com baixa renda, sendo destes, $48 \%$ são dependentes de apenas um salário mínimo.

Quanto aos aspectos clínicos (Tabela 2), observou-se uma predominância de indivíduos que sofreram a lesão por acidentes automobilísticos (60\%). O nível torácico (52\%) foi o mais atingido, seguido de cervical (36\%) e lombar (12\%), 
resultando em paraplegia, que teve $60 \%$ dos casos com grau de lesão incompleta (72\%).

Tabela 2. Dados clínicos das pessoas com TME.

\begin{tabular}{ccc}
\hline Variáveis & $\begin{array}{c}\text { Frequência absoluta } \\
(\mathbf{n})\end{array}$ & $\begin{array}{c}\text { Frequência relativa } \\
\mathbf{( \% )}\end{array}$ \\
\hline Etiologia do trauma & 3 & 12 \\
Arma de fogo & 2 & 8 \\
Queda & 15 & 60 \\
Acidente automobilístico & 5 & 20 \\
Outros & 7 & 28 \\
Grau da lesão & 18 & 72 \\
Completa & & \\
Incompleta & 8 & 32 \\
Tempo de internação hospitalar & 6 & 24 \\
Até 15 dias & 6 & 24 \\
Até 30 dias & 5 & 20 \\
Até 60 dias & & \\
> 60 dias & 16 & 64 \\
Tempo de lesão & 7 & 28 \\
1 a 3 anos & 2 & 8 \\
4 a 10 anos & & \\
> 10 anos & 9 & 36 \\
Cervical & 13 & 52 \\
Torácica & 3 & 12 \\
Lombar & &
\end{tabular}

A maior parte da amostra tinha entre um e três anos de lesão (64\%), e a média de tempo de internação hospitalar foi de até quinze dias (32\%).

A partir dos resultados obtidos por meio do questionário WHOQOL-BREF foi possível avaliar a qualidade de vida (QV) das pessoas com traumatismo da medula espinal. Na Figura 1 estão apresentados os valores em porcentagem da qualidade de vida geral, ou seja, o percentual dos valores obtidos nos domínios físicos, psicológicos, relações sociais e ambientais. A maioria dos indivíduos (72\%) avaliaram sua 
qualidade de vida geral como regular e nenhum com resultado de muito boa.

Quando foi feito a correlação da qualidade de vida dos indivíduos com as variáveis sociodemográficas, não foi identificada nenhuma correlação significante.

Figura 1. Percentual dos entrevistados quanto a qualidade de vida geral.

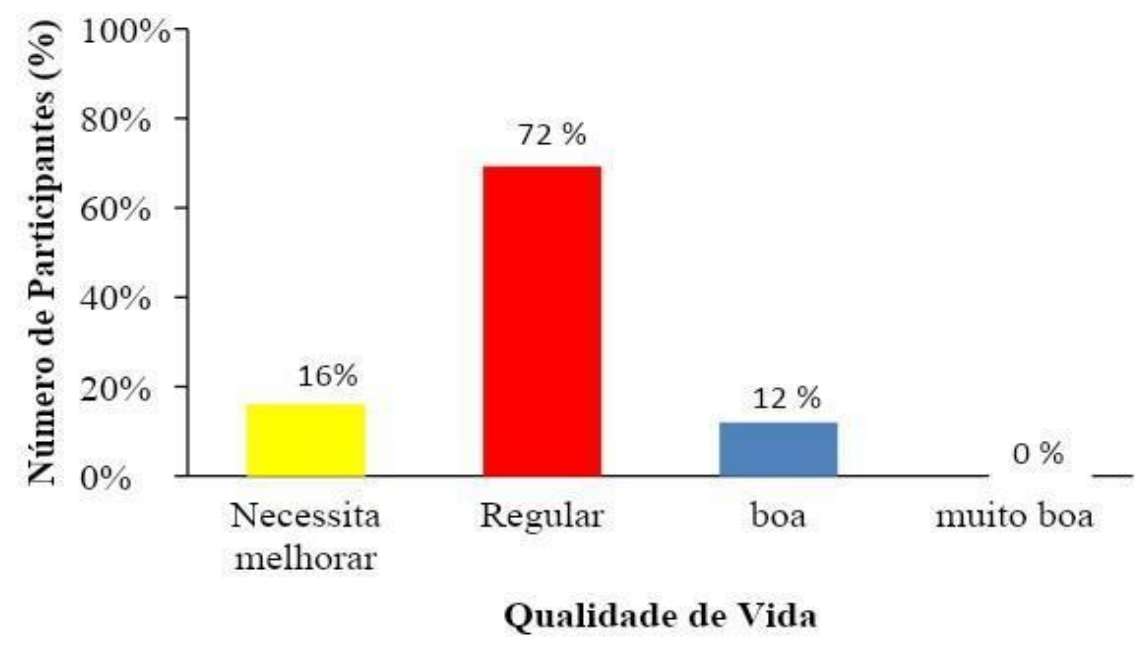

\section{DISCUSSÃO}

O número de trauma ser maior em homens se dá pelo fato deles estarem mais expostos ao risco de acidentes ${ }^{8}$. Muitos destes acidentes estão relacionados às festividades, combinado ao uso de drogas ilícitas e lícitas em geral ${ }^{9}$.

O fato de adultos jovens serem mais afetados justifica eles estarem fora do mercado de trabalho e serem dependentes de benefícios públicos, por não conseguirem voltar a exercer suas funções trabalhistas após a lesão ${ }^{10}$.

De acordo com os dados levantados grande parte dos pacientes que sofreram TME tem ensino fundamental e 
médio, e esse quesito é uma das barreiras mais frequentes na inserção dessa população no mercado de trabalho. 0 impacto socioeconômico é evidente, e como o estudo mostra a prevalência de pessoas com baixa renda, $48 \%$ da população dependentes de apenas um salário mínimo, isso acontece, pois, os mais afetados foram jovens que se encontravam no auge de sua inserção econômica, ficando dependentes de benefício previdenciário mínimo propiciado pelo governo federal ${ }^{11}$.

A maior prevalência da causa do trauma foram os acidentes automobilísticos ${ }^{9}$. Esse elevado índice de lesão por acidente, muitas vezes ocorre pelo grande consumo de bebidas alcoólicas, excesso de velocidade e uso inadequado de cinto de segurança ${ }^{12}$.

O nível da coluna mais atingido foi a torácica, similar a outros estudos ${ }^{10,11}$, o que resulta em pacientes com paraplegia de grau incompleta. Acredita-se que a diminuição de lesões completas tenha reduzido devido ao preparo que as equipes de resgate estão tendo e por um melhor atendimento de emergência ${ }^{13,14}$.

O período de internação hospitalar tem diminuído, pois esses pacientes recebem alta hospitalar assim que estão estáveis e iniciam a reabilitação nos centros especializados ${ }^{15}$.

O questionário WHOQOL-BREF tem como resultado geral de 1 a 5 pontos, sendo que 1 a 2,9 o paciente tem necessidade de melhora, 3 a 3,9 é considerado regular, 4 a 4,9 é boa e 5 pontos considerado qualidade de vida muito boa ${ }^{16}$. É importante conhecer em quais aspectos os 
indivíduos estão satisfeitos ou insatisfeitos, através do WHOQOL-BREF é possível analisar a qualidade de vida pelos seus domínios físicos, psicológicos, relações sociais e ambientais.

No domínio físico os indivíduos relataram ter qualidade de vida regular, pois sentem muita dor, falta de capacidade de locomoção, fadiga e cansaço, tem dependência de remédios e afeta o sono. A dor acontece após o trauma, consequência da espasticidade, hipersensibilidade ao toque, e isso atrapalha na locomoção e atividade recreativas ${ }^{18,19}$.

Alguns indivíduos buscam apoio em crenças pessoais e religiosas, onde conseguem aceitar sua aparência física e ter autoestima, porém, outros têm sentimentos negativos, não aceitam sua imagem corporal e apresentam déficit de memória e concentração ${ }^{17}$.

O domínio de relações sociais demonstra o suporte familiar e de amigos, que apresenta bons resultados, e resultados insatisfatórios com a vida sexual após a lesão ${ }^{17-}$ 19.

O domínio de meio ambiente demonstra insatisfação, que se dá pela falta de dinheiro dos indivíduos, a falta de oportunidade de lazer e atividades recreativas, falta de infraestrutura de moradia e transporte. A acessibilidade é a principal barreira para as pessoas com traumatismo da medula espinal, e a dificuldade de usarem os serviços de saúde ${ }^{17}$. 
A qualidade de vida um nome próprio para o bem-estar das pessoas e o ambiente em que vivem que torne o sujeito feliz e satisfeito, no entanto, os desejos raramente atingem um estado de completa satisfação, a não ser que em um curto período, o conceito de qualidade de vida não só varia de pessoa para pessoa como também de lugar e tempo ${ }^{20}$.

\section{CONCLUSÃO}

Conclui-se nesse estudo que a qualidade de vida (QV) das pessoas com traumatismo da medula espinhal é regular e quando comparada com algumas variáveis sociodemográficas não houve correlação estatística significativa, demonstrando que a QV não depende da idade, funcionalidade e tempo de lesão, levando em consideração que QV é algo subjetivo e individual.

Talvez a utilização de novos instrumentos de avaliação para essa população possa contribuir para uma melhor verificação de qualidade de vida após a lesão.

\section{AGRADECIMENTOS}

Agradeço às famílias e pacientes por sua participação neste estudo, aos fisioterapeutas do Centro Especializado em Reabilitação (CER) onde a coleta de dados para o estudo foi realizada em parceria com a Universidade Católica Dom Bosco (UCDB) de Campo Grande-MS. 


\section{REFERÊNCIAS}

1.Venturini DA, Decésaro MN, Marcon SS. Alterações e expectativas vivenciadas pelos indivíduos com lesão raquimedular e suas famílias. Ver Esc Enferm USP 2007;41:589-96.

https://doi.org/10.1590/S0080-62342007000400008

2.Silva AR, Santos JAT, Barros JF, Gorla JI. Qualidade de vida e independência funcional de lesados medulares. Revista Eletrônica Gestão \& Saúde 2013;4:2151-64.

https://periodicos.unb.br/index.php/rgs/article/view/248

3. Ministério da Saúde (endereço na Internet). Brasil: Secretaria de Atenção à Saúde. Departamento de Ações Programáticas Estratégicas. Diretrizes de atenção à pessoa com lesão medular. Brasília: Ministério da Saúde, 2013.2 Disponível em: http://bvsms.saude.gov.br/bvs/publicacoes/diretrizes atencao pesso a lesao medular.pdf

4.Coura AS, França ISX, Enders BC, Barbosa ML, Souza JRS. Incapacidade funcional e associações com aspectos sociodemográficos em adultos com lesão medular. Rev Lat Am Enfer 2012;20:1-9. https://doi.org/10.1590/S0104-11692012000100012

5.American Spinal Injury Association (ASIA) (endereço na Internet). International Standarts for neurological and functional classification of spinal cord injury. Chicago: American Spine Injury Association, 2015 (acessado em: 20/08/2016). Disponível em: http://asiaspinalinjury.org/

6. Organização Mundial De Saúde (OMS) (endereço na Internet). Programa de Saúde Mental. Versão em português dos instrumentos de avaliação de qualidade de vida (WHOQOL). Departamento de Psiquiatria e Medicina Legal da Universidade Federal do Rio Grande do Sul, 1998 (acessado em: 20/07/2016). Disponível em: http://www.ufrgs.br/psiquiatria/psiq/whogol84.html

7. Bampi LNS, Guilhem DL, David D. Qualidade de vida em pessoas com lesão medular traumática: um estudo com o WHOQOL-bref. Rev Bras Epidemiol 2008;11:67-77. https://doi.org/10.1590/S1415790X2008000100006

8.Goncalves AMT, Rosa LN, D'angelo CT, Savordelli CL, Bonin GL, Squarcino IM, et al. Aspectos epidemiológicos da lesão medular traumática na área de referência do Hospital Estadual Mario Covas. Arqmedabc 2007;32:6-64.

https://www.portalnepas.org.br/amabc/article/view/183/179

9.Rodrigues AV, Vidal WAS, Lemes JÁ, Gôngora CS, Neves TC, Santos SMS, et al. Estudo sobre as características da dor em pacientes com lesão medular. Acta Fisiatr 2012;19:7-171. https://doi.org/10.5935/0104-7795.20120027

10.Santiago LMM, Barbosa LCS, Guerra RO, Melo FRLV. Aspectos sociodemográficos e clínicos de homens com lesão medular traumática em um centro urbano do nordeste brasileiro. Arq Bras Ciênc Saúde 2012;37:137-42.

https://www.portalnepas.org.br/abcs/article/view/27/28 
11.Brito LMO, Chein MBC, Marinho SC, Duarte TB. Avaliação epidemiológica dos pacientes vítimas de traumatismoraquimedular. Rev Col Bras Cir 2011;38:304-9. https://doi.org/10.1590/S010069912011000500004

12.Santos RA, Almeida MLO, Silva MF. Perfil clínico e epidemiológico de pacientes com traumatismo raquimedular. Fisioter Bras 2013;14:16. http://dx.doi.org/10.33233/fb.v14i3.394

13. Rabeh S, Caliri MHL. Capacidade funcional em indivíduos com lesão de medula espinhal. Acta Paul Enferm 2010;23:7-321. http://dx.doi.org/10.1590/S0103-21002010000300002

14.Gaspar AP, Ingham SJM, Vianna PCP, Santos FPE, Chamlian TR, Puertas EB. Avaliação epidemiológica dos pacientes com lesão medular atendidos no Lar Escola São Francisco. Acta fisiátr 2003;10:73-7. http://www.journals.usp.br/actafisiatrica/article/view/102444/10076 1

15.Custódio NRO, Carneiro MR, Feres CC, Lima GHS, Jubé MRR, Watanabe LE, et al. Lesão medular no Centro de Reabilitação e Readaptação Dr. Henrique Santillo (CRER- GO). Coluna/Colunna 2009; 8:265-8. https://doi.org/10.1590/S1808-18512009000300005

16.Bampi LNS. Percepção de qualidade de vida de pessoas com lesão medular traumática: uma forma de estudar a experiência da deficiência. (Tese) Brasilia: Universidade de Brasília, 2007. http://repositorio.unb.br/handle/10482/5810

17. Corrêa LS. Estudo de qualidade de vida em pessoas com lesão medular (Dissertação). Manaus: Universidade Federal do Amazonas, 2011.http://tede.ufam.edu.br/bitstream/tede/3663/4/Lionela\%20Cor rea.pdf

18. França ISX, Coura AS, França EG, Basílio NNV, Souto RQ. Qualidade de vida de adultos com lesão medular: um estudo com WHOQOL-bref. Ver Esc Enferm USP 2011;45:71-1364. https://doi.org/10.1590/S0080-62342011000600013

19.França ISX, Coura As, Sousa FS, Almeida PC, Pagliuca LMF. Qualidade de vida em pacientes com lesão medular. Rev Gaúcha Enferm 2013;34:155-63. https://doi.org/10.1590/S198314472013000100020

20.Santos JAT. Qualidade de vida e independência funcional de lesados medulares (Dissertação). Brasilia: Universidade de Brasília, Faculdade de Educação Física, 2010.

http://repositorio.unb.br/handle/10482/6549 